\title{
Cerebellar hemorrhage following endoscopic third ventricular colloid cyst decompression
}

Sir,

Cerebellar hemorrhage following supratentorial surgery is a rare complication with only 29 cases 
reported in the literature. ${ }^{[1-3]}$ However, there has been no reported case of cerebellar hemorrhage following endoscopic surgery.

We present the case of a patient aged 45 years, a known hypertensive and hypothyroid on treatment developed features suggestive of raised intracranial pressure. On examination there were no focal neurological deficits. Computed tomography (CT) was suggestive of a colloid cyst [Figure 1].

He underwent endoscopic fenestration and decompression of cyst. The cyst contents were drained completely but the capsule was partially excised due to the dense adhesions with thalamostriate vein. The surgery was uneventful. Five hours after surgery patient suddenly became drowsy with shallow respiration. He was intubated and underwent an emergency external ventricular drainage. The computed topography (CT) showed a well-organized hematoma measuring $5 \times 2.7 \times 2.5 \mathrm{~cm}$ in the left cerebellar lobe with no mass effect [Figure 2]. The coagulation profile was normal as were the routine biochemical and hematological parameters. The patient's blood pressure was normal. In view of the normal neurological status and clot volume of $17 \mathrm{ml}$ it was decided to continue with the conservative management.

A repeat CT done on postoperative day 7 showed a persisting hematoma with no change in size. However, in view of persistent headache along with surrounding edema, hydrocephalus along with effacement of basal cisterns and fourth ventricle, a decision to evacuate the hematoma was taken. A left paramedian suboccipital craniectomy and evacuation of hematoma was done along with a ventriculoperitoneal (VP) shunt in the same sitting. Patient was discharged in normal neurological status with no motor sensory deficits.

Patient developing remote infratentorial hematoma after supratentorial surgery is a rare complication with only 29 cases available in the reported literature. ${ }^{[1-3]}$ We did an extensive literature review including our own experience of 96 cases with endoscopic decompression of colloid cyst but could not find a similar case. ${ }^{[4,5]}$

The mechanism of cerebellar hemorrhage after supratentorial surgeries is a matter of debate and is probably multifactorial. Various factors have been proposed which include the following causes.

Intracranial hypotension due to sudden decompression of the ventricles might lead to a cerebellar hematoma, as there could exist a pressure differential between the two compartments. Other predisposing factors suggested include an altered coagulation profile, ${ }^{[1]}$ (but most reported cases including ours had a normal coagulation profile) and postoperative hypertension. An important proposed factor is overdrainage of cerebrospinal fluid (CSF) intraoperatively or postoperatively via drains. ${ }^{[3]}$ In all the patients, the occurrence of hemorrhage was associated with loss/removal of large amounts of CSF either intraoperatively or postoperatively. Another postulated cause has been excessive rotation with hyperextension of head leading to obstruction of internal jugular veins. ${ }^{[3]}$

In our patient, the plausible mechanism could be a combination of sudden supratentorial decompression leading to intracranial hypotension with resulting displacement of the cerebellum. Various management strategies have been suggested but the most important indicators for surgery include the neurological status of the patient, the presence of hydrocephalus, the size of the hematoma and the extent of fourth ventricular and brainstem compression. The reported mortality and morbidity has been high (33 and 58\%).

\section{Hitesh Gurjar, Srinivas Dwarakanath, Bhavani Shankar Sharma, Sarat Chandra}

Department of Neurosurgery, All India Institute of Medical Sciences, New Delhi, India.

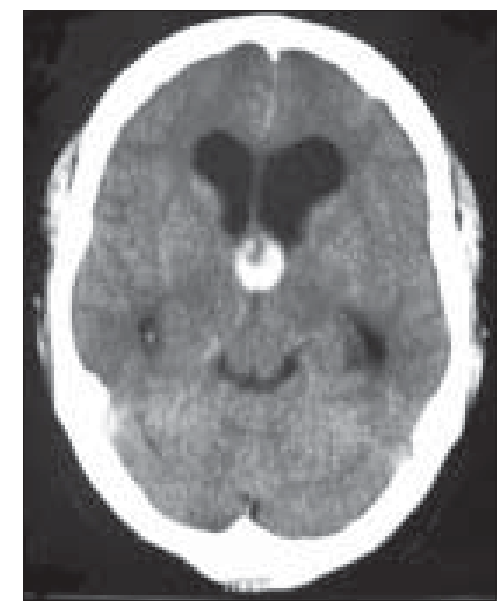

Figure 1: Preoperative CT scan of the head showing a Colloid cyst

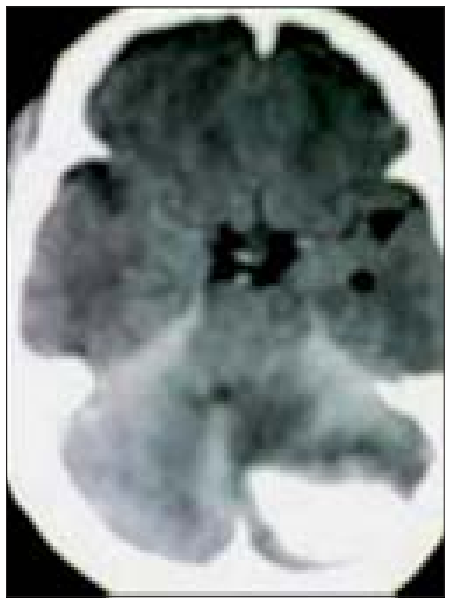

Figure 2: Postoperative CT scan of the head showing a left cerebellar hematoma 


\section{References}

1. Kaplan SS, Lauryssen C. Cerebellar hemorrhage after evacuation of an acute supratentorial subdural hematoma. Br J Neurosurg 1999;13:329-31.

2. Miranda P, Alen JF, Rivas J, Perez A, Ramos A. Cerebellar hematoma following transsphenoidal surgery. Acta Radiol 2005;46:184-6.

3. Yoshida S, Yonekawa Y, Yamashita K, Ihara I, Morooka Y. Cerebellar hemorrhage after supratentorial craniotomy--report of three cases. Neurol Med Chir (Tokyo) 1990;30:738-43.

4. Longatti P, Godano U, Gangemi M, Delitala A, Morace E, Genitori L, \& al. Cooperative study by the Italian neuroendoscopy group on the treatment of 61 colloid cysts. Childs Nerv Syst 2006;22:1263-7.

5. Rodziewicz GS, Smith MV, Hodge CJ Jr. Endoseopic colloid cyst surgery. Neurosurgery 2000;46:655-62.

Accepted on 25-09-2007 